\title{
Online evaluation of novel choices by simultaneous representation of multiple memories
}

\author{
Helen C Barron ${ }^{1}$, Raymond J Dolan ${ }^{1}$ \& Timothy E J Behrens ${ }^{1,2}$ \\ Prior experience is critical for decision-making. It enables explicit representation of potential outcomes and provides training \\ to valuation mechanisms. However, we can also make choices in the absence of prior experience by merely imagining the \\ consequences of a new experience. Using functional magnetic resonance imaging repetition suppression in humans, we examined \\ how neuronal representations of novel rewards can be constructed and evaluated. A likely novel experience was constructed by \\ invoking multiple independent memories in hippocampus and medial prefrontal cortex. This construction persisted for only a \\ short time period, during which new associations were observed between the memories for component items. Together, these \\ findings suggest that, in the absence of direct experience, coactivation of multiple relevant memories can provide a training \\ signal to the valuation system that allows the consequences of new experiences to be imagined and acted on.
}

Humans display remarkable flexibility in their behavior. Like other animals, we guide our behavior through direct experience, but we can also infer the likely consequences of actions that have never been taken ${ }^{1,2}$. Through generalizing principles and applying them to new situations ${ }^{3,4}$, we can predict new relationships and statistical structures in our environment and use these to estimate the value of new events ${ }^{1,5,6}$. Although some progress has been made in uncovering the brain regions that underlie these complex abilities ${ }^{1,3-7}$, little or no progress has been made in understanding how neuronal networks support these complex computations, partly because it is unclear to what extent such computations exist in species in which we can readily measure single-cell activity.

One potential mechanism that allows for upcoming events to be evaluated involves using past experience to predict consequences of future possible scenarios. In rodents, hippocampal firing sequences at choice points predict or 'preplay' the forthcoming environment ${ }^{8}$, and the likely outcomes of their decision can later be decoded in the orbitofrontal cortex ${ }^{9}$. In contrast, when choosing between novel options, there is no direct experience from which to preplay and evaluate future options. However, it is possible that the representation of an upcoming novel outcome may be constructed by combining multiple distinct relevant experiences, preplayed simultaneously.

To test these predictions, we required access to the information content of neural populations underlying the representation of a novel experience. Despite the poor spatial resolution of functional magnetic resonance imaging (fMRI), there are well-validated strategies that can reveal underlying cellular representations. For example, fMRI adaptation takes advantage of the fact that activated cellular ensembles in a voxel show a relative suppression in their activity in response to repetition of a stimulus to which they recently responded. Despite ambiguity in the biophysical mechanism underlying repetition suppression $^{10,11}$, when combined with careful experimental design the technique allows for inferences to be made about the underlying neuronal representations ${ }^{12,13}$.

We used fMRI adaptation to probe the neural representation of a novel food reward. We hypothesized that, if the representation of a novel food was constructed by explicit combination of multiple distinct experiences, we would observe fMRI adaptation when subjects evaluated a novel reward immediately after evaluating a component ingredient. Furthermore, if multiple experiences were replayed simultaneously, plasticity might result between the underlying neuronal assemblies. Hence, experiences used to construct the same novel good would later adapt to each other. Lastly, we hypothesized that this complex construction process would not be required after an independent neuronal representation of the novel good had been established. We should therefore observe a reduction in each adaptation effect after allowing the subjects to either experience the novel good directly or simulate the novel good repeatedly. This repetition suppression procedure allowed us to probe the neural mechanisms that underlie human capacity for flexible, online, value construction.

\section{RESULTS}

\section{Deciding between novel goods}

We created 13 novel goods whose values were unknown to the subjects (Fig. 1). However, each good was a novel combination of two different familiar foods (Fig. 1a). Participants were given the opportunity to observe these novel goods without being allowed to sample them by either taste or smell.

To first establish that these goods activate known value-related brain regions, we measured fMRI activity in 19 subjects while they evaluated and chose from pairs of these novel goods (Fig. 1b). After the scan session, subjects performed a Becker-DeGroot-Marschak (BDM) auction ${ }^{14}$ that allowed us to measure subjects' constructed value for each good. Consistent with reports in simpler valuation

${ }^{1}$ The Wellcome Trust Centre for Neuroimaging, Institute of Neurology, University College London, London, UK. ${ }^{2}$ The Oxford Centre for Functional Magnetic Resonance
Imaging of the Brain, University of Oxford, Oxford, UK. Correspondence should be addressed to H.C.B. (helen.barron.10@ucl.ac.uk) or T.E.J.B. (behrens@fmrib.ox.ac.uk).

Received 5 June; accepted 9 August; published online 8 September 2013; doi:10.1038/nn.3515 
Figure 1 Experimental design. (a) We made 13 novel goods from the combination of two familiar food types that had not previously been tasted together. Two examples are shown here: avocado and raspberry smoothie $(A B)$ and tea-jelly (CD). (b) Participants made binary decisions between the novel goods while in the scanner. (c) Prior to entering the scanner, two of the novel goods were chosen for each participant. Participants learned to associate each of these novel goods and their respective components with two abstract stimuli. (d) In the scanner, participants vividly imagined the sensory properties of the food items in response to each abstract stimulus presented.

contexts, we observed a signal that correlated with the value of the chosen option in a network of brain regions that included ventral and dorsal medial prefrontal cortex (vmPFC and $\mathrm{dmPFC}$, respectively), and posterior cingulate cortex (mPFC: $P=0.001$ familywise error (FWE) corrected on cluster level, peak $t_{17}=6.30$; Fig. 2a). The involvement of both vmPFC and dmPFC is of particular interest given that the task required subjects to construct and evaluate a model of a future outcome. This involvement accords with recent evidence that vmPFC encodes value preference for executable choices and dmPFC does so for choices that are modeled abstractly ${ }^{7}$.

To evaluate these novel goods, subjects could not rely on pre-learnt values. Thus, their only recourse was to construct, online, an expectation of the compound's value from knowledge of the individual components. A key question is whether subjects constructed a novel representation of the compound by explicitly combining the representations of each component and, if so, which brain regions support this construction process. We reasoned that this construction process could be measured using fMRI adaptation. Activity relating to the construction of the compound value would be suppressed when preceded by a related component if, and only if, the subject had engaged the neuronal ensembles of the components when constructing a representation of the compound.

\section{Constructing representations of novel goods using memories}

For every participant, we selected 2 of the 13 novel compounds, here referred to as $\mathrm{AB}$ and $\mathrm{CD}$, each consisting of two familiar individual components ( $\mathrm{A}$ and $\mathrm{B}, \mathrm{C}$ and $\mathrm{D}$ ) that subjects had tasted immediately before the experiment (Fig. 1a). To avoid visual confounds in a later analysis, we trained subjects to associate each of the six component and compound foods (A, B, C, D, AB and CD) with two different abstract shapes (Fig. 1c). Participants trained extensively on these associations between food items and abstract shapes. In the final block of trials, the mean accuracy was $97.8 \%$, with a mean reaction time of $845.2 \mathrm{~ms}$.

On each trial in the scanner, we presented a distinct shape that served as an instruction cue for subjects to elicit an explicit mental representation of the associated food (Fig. 1d). The key comparison of interest here was the brain activity elicited by novel goods when preceded by related components (for example, A or B followed by AB) compared with novel goods when preceded by unrelated components (for example, C or D followed by AB).

Early in the experiment (block 1 of 3), we observed fMRI adaptation between the representation of novel goods and their constituent components in both mPFC $(P<0.001$, FWE corrected on cluster level, peak $t_{18}=4.45$; Fig. $\left.2 \mathbf{b}\right)$ and bilateral hippocampus $\left(t_{18}=2.55\right.$, $P=0.010$ using region of interest (ROI) analysis; Online Methods and Fig. 2b). These two brain regions are components of a network that is commonly activated in studies of value ${ }^{7,15-18}$, episodic memory $y^{4,19,20}$ and spatial navigation ${ }^{12}$. Our result implies that these brain regions construct a value representation of a novel item from component memories, and do so by simultaneously engaging neuronal representations of these components.

\section{Plasticity between simultaneously active memories}

If this is the case then it follows that during the construction of the compound good $\mathrm{AB}$, the neuronal ensembles representing components $A$ and $B$ should be simultaneously active. We reasoned that this simultaneous activity, which first occurred during the stimulus-item training phase before scanning, would induce experience-dependent plasticity between cellular elements in these two ensembles-a plasticity evident in the scanning trials as a shadow of this value construction process. For example, after constructing a representation of 'tea-jelly dessert', we reasoned that cellular representations of tea would induce activity in jelly-preferring ensembles and vice versa. This can also be tested using fMRI adaptation, which predicts a differential effect for components that were part of the same compound compared with components that were not. Indeed, when we compared early trials of A that were preceded by $\mathrm{B}$ to those that were preceded by $\mathrm{C}$, we again found relative suppression in $\mathrm{mPFC}$ activity $(P=0.014$, FWE corrected on cluster level, peak $t_{18}=4.24$; Fig. $2 \mathrm{c}$ ), but not hippocampus ( $t_{18}=0.34, P=0.367$ using ROI analysis, Online Methods).

Notably, across all three blocks, the extent to which individual participants showed adaptation between related components in $\mathrm{mPFC}$, and in the hippocampus, was predicted by the average value of the novel items (mPFC, $r=0.47$ and $P=0.040$; hippocampus, $r=0.58$ and $P=0.010$ ), but not component items (mPFC, $r=-0.05, P=0.833$; hippocampus, $r=-0.09, P=0.730$ ). This suggests that the mechanism underlying this suppression occurred during the earlier construction of the novel good and not during the participant's elicitation of the component item at the time that this signal was measured. Indeed, in both structures, the correlation with the value of the novel good survived the removal of any signal attributable to the component values 
Figure 2 Neural correlates of constructing and evaluating a novel good. (a) While participants made binary choices between novel goods, the mPFC (extending into dmPFC) encoded chosen value. (b) The mPFC and hippocampus showed repetition suppression to a novel good when preceded by a related component (for example, tea-jelly preceded by tea) compared with when preceded by an unrelated component (for example, tea-jelly preceded by avocado). (c) The MPFC showed repetition suppression to a component food item when preceded by the related component (for example, tea preceded by jelly) compared with when preceded by an unrelated component (for example, tea preceded by avocado). (d,e) In mPFC and hippocampus, a significant positive correlation was revealed between the amount of suppression between related components (across all blocks) and the average value participants assigned to the novel goods (after removing effects attributable to the value of the components; for mPFC: $r=0.51$, $P=0.015$; hippocampus, $r=0.60, P=0.004$ ), respectively. (f) Both adaptation effects showed comparable effect size across the ventral-todorsal gradient of mPFC (mean \pm s.e.m. across participants). The locations of the ROIs are shown and the effect size for both adaptation measures was scaled such that the peak value was equal to 1. There was no significant difference between the two adaptation effects at any point on this gradient $(P>0.300$ for all ROIs).
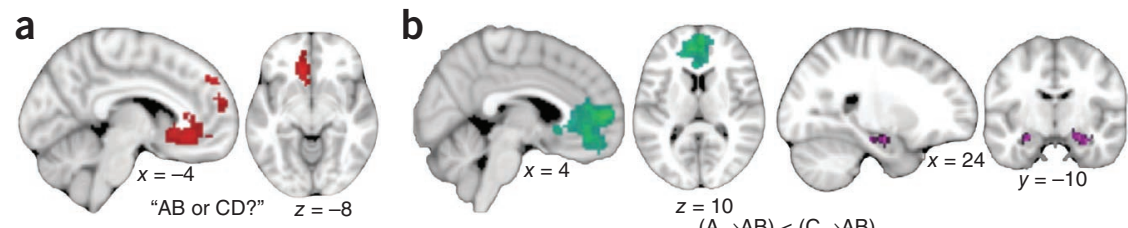

C
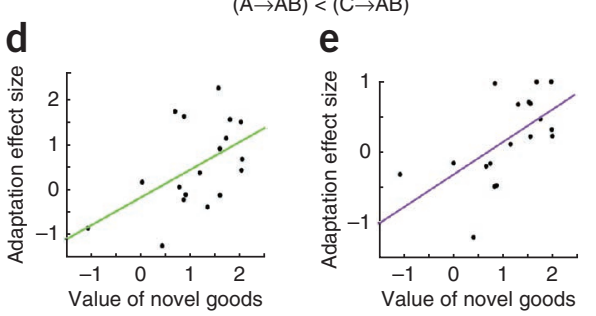
Value of novel goods Value of novel goods

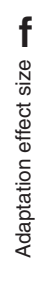

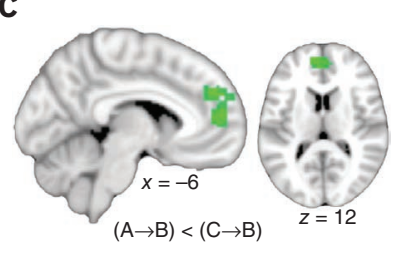

f

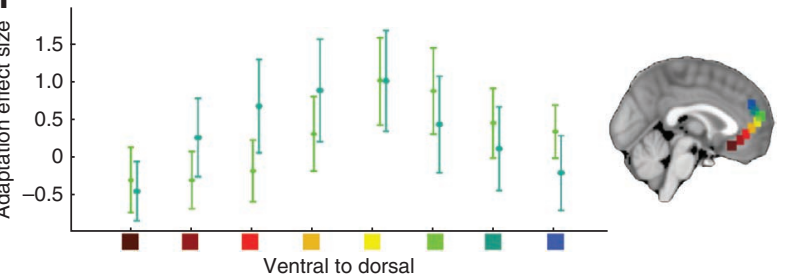

(mPFC, $r=0.51, P=0.015$, Fig. 2d; hippocampus, $r=0.60, P=0.004$; Fig. 2e and Supplementary Fig. 1). Together, these findings support value-dependent plasticity in related components as a consequence of coactivation during construction of the novel goods.

It is important to note that these three de facto tests of mPFC function (valuation, construction and plasticity) do not rely on the same data. Despite slight differences in thresholded peak locations of the two adaptation effects, they showed similar patterns of activity in mPFC (Fig. 2f). mPFC can therefore evaluate novel goods by constructing explicit representations of expected outcomes from familiar components, a process that engenders plasticity between simultaneously active component representations.

\section{The influence of sensory experience upon construction}

We then asked whether consummatory exposure to the novel goods would reduce a need to construct value online. To test this idea, we repeated the experiment in a second group of 20 subjects with one important difference. This second group (familiar) was given a single sample of each of the 13 novel compound goods to taste before the experiment. Notably, both groups underwent the same item-stimulus learning task before entering the scanner, and there was no significant difference between groups in reaction time or accuracy on the final block of trials during the learning task $(P>0.150$; Supplementary Table 1). Any difference between the two groups in the representation or evaluation of novel goods could therefore be attributed to the effect of sensory exposure.

We first assessed value effects during decision trials. Both groups showed similar consistency in their choices (Supplementary Fig. 2). As was the case for the unfamiliar group, the familiar group encoded chosen value activity in a network of value-related brain regions that included $\mathrm{mPFC}$ (Fig. 3a). In both groups, the neural activity observed in $\mathrm{mPFC}$ was consistent with a role for this brain region in the evaluation of compound goods (Fig. 3b,c).

To determine whether this single experience was enough to reduce a need for online value construction, we compared adaptation effects across the two groups. To avoid selection bias, we used ROIs derived from whole-brain adaptation effects averaged across both adaptation contrasts in the two groups (Online Methods and Fig. 4a,b). A between-group comparison in these ROIs revealed significant differences in the adaptation effects between the familiar and unfamiliar participants in both $\mathrm{mPFC}$ (group $\times$ condition interaction, $P=0.018, F_{1,144}=5.76$, three-way ANOVA, Online Methods) and hippocampus (group $\times$ adaptation type $\times$ condition interaction, $P=0.035, F_{1,144}=4.52$, three-way ANOVA, Online Methods) .
Figure 3 Sensory exposure to a novel good: comparison between the unfamiliar and familiar groups during the decision-making task. (a) In the familiar group, the mPFC correlated with chosen value during the decision-making task (thresholded at $P<0.01$, uncorrected for visualization). (b) ROI used to assess value signals in both groups of participants during the decision task. (c) During the decision-making task, the unfamiliar and familiar groups showed comparable chosen value signals in mPFC (left, average of all task blocks for each group), and in the unfamiliar group there was no change in the chosen value signal across time (right, block 1 versus blocks 2 and 3). Parameter estimates were extracted from ROI shown in $\mathbf{b}$ (mean \pm s.e.m. across participants). a.u., arbitrary units. a

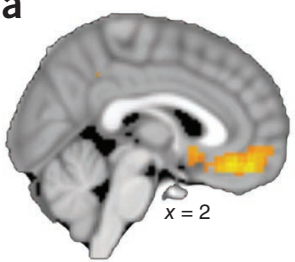

b

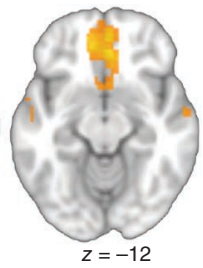

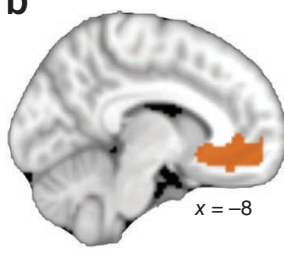

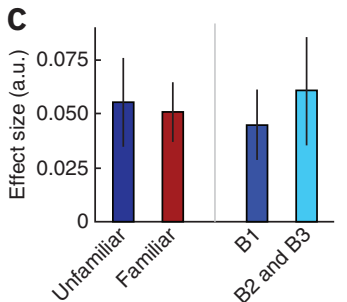


Figure 4 Sensory exposure to a novel good: comparison between the unfamiliar and familiar groups during construction of a novel good. (a) ROI used to compare mPFC adaptation effects. (b) ROI used to compare hippocampus adaptation effects. (c) In mPFC, the familiar group showed less adaptation between the novel goods and their related components (left, $P=0.053$, trend) and significantly less adaptation between related components (middle, $P=0.033$; both extracted from the ROI shown in a). In the hippocampus, the familiar group showed significantly less adaptation between the novel goods and their related components (right, $P=0.008$, extracted from ROIs shown in b).

${ }^{*} P<0.05$. Data are presented as mean \pm s.e.m. across participants.

Using post hoc two-sample $t$ tests to decompose these interactions, we found that, relative to the unfamiliar group, the familiar group showed reduced adaptation between the novel goods and their related components in mPFC (group difference: trend, $t_{18}=1.70, P=0.053$; Fig. 4c) and in the hippocampus (group difference: $t_{18}=3.11$, $P=0.003$; Fig. 4c). Furthermore, the familiar group did not show plasticity in $\mathrm{mPFC}$ between the representation of the constituent components of a novel good (group difference: $t_{18}=1.96, P=0.033$, Fig. 4c). Crucially, there was no significant difference between groups in their ability to accurately elicit the correct representations during the imagination task (group comparison of accuracy, $P=0.82$; reaction time, $P=0.89$ ) or in the average subjective value assigned to any of the novel goods used in the adaptation task $(P>0.05$ for all assigned novel goods; Supplementary Fig. 3). This result therefore suggests that even a single previous experience of a good is sufficient to reduce a requirement for online value construction. This is particularly notable given that extensive experience is required to reduce goal-oriented behavior and establish habitual actions ${ }^{21}$.

\section{Temporal dynamics of the construction mechanism}

If experiential and constructed valuation use distinct neural mechanisms, it is possible that the value construction mechanism could itself substitute for a direct experience and train experiential valuation mechanisms. As the experiment progressed, subjects gained substantial experience in constructing the representation of the novel good. We asked whether, after multiple previous simulations of an experience, it was still necessary to construct and evaluate the representation of novel goods anew on each trial. Alternatively, were values learned despite participants never having experienced the novel good? As our experiment extended over three separate blocks, we were able to study changes in value construction-related adaptation effects over time (Figs. 5 and 6). a
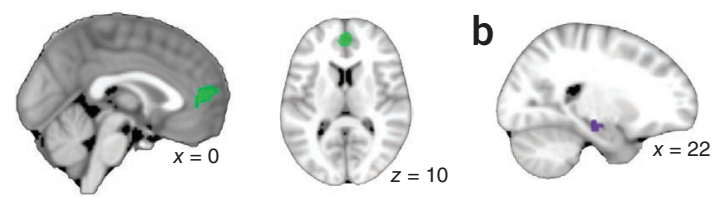

C

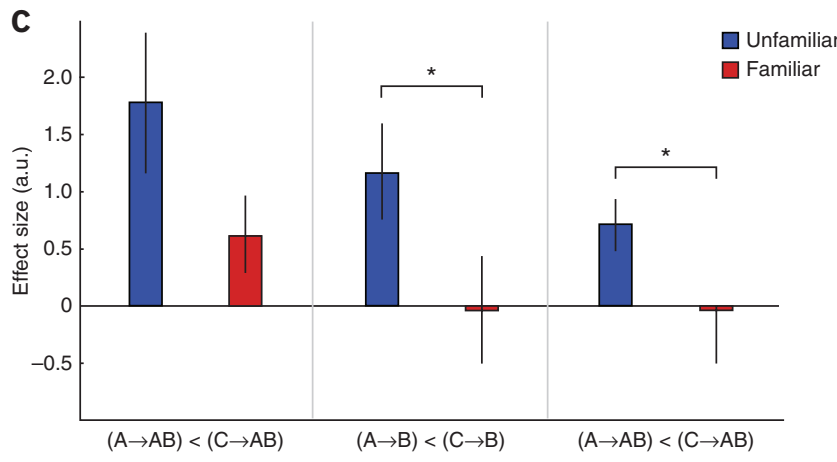

Previous studies have found that goal-directed choice mechanisms exhibit marked differences early and late in choice experiments ${ }^{17}$. We used a three-way ANOVA (Online Methods) to identify attenuation of adaptation effects in $\mathrm{mPFC}$ and hippocampus in the unfamiliar group across the scanning session (block $\times$ condition interaction for mPFC, $P=0.004, F_{1,144}=8.44$; block $\times$ adaptation-type interaction for hippocampus, $P=0.011, F_{1,144}=6.56$ ). Post hoc $t$ tests comparing block 1 with all remaining blocks revealed a significant reduction in adaptation over time of a novel good to its related component ( $\mathrm{mPFC}$, $t_{18}=2.12, P=0.024$; hippocampus, $t_{18}=2.13, P=0.024$; Fig. 5a) and in the plasticity between related components (mPFC, $t_{18}=1.85$, $P=0.041$; but not hippocampus, $t_{18}=0.81, P=0.785$; Fig. 6a).

To ensure that sensitivity to the construction process was maintained across the duration of the experiment, we also considered temporal dynamics of other adaptation effects and of value signals encoded on decision trials. In the unfamiliar group, both adaptation in $\mathrm{mPFC}$ to repetition of any item (but not stimulus) and adaptation in visual areas to repetition of a stimulus did not show a reduction over time (one-tailed paired $t$ tests, $t_{18}=0.46, P=0.326$; Fig. 5a; $t_{18}=0.50, P=0.312$; Supplementary Fig. 4a). Furthermore, the chosen value signal encoded by $\mathrm{mPFC}$ also did not reduce over time, but instead remained consistent across sessions (Fig. 3c). In addition, performance on the imagination task improved across blocks (Fig. 5b,c). Rather than a loss of sensitivity, this suggests that the diminishing adaptation effects demonstrate that simulated experience is sufficient a

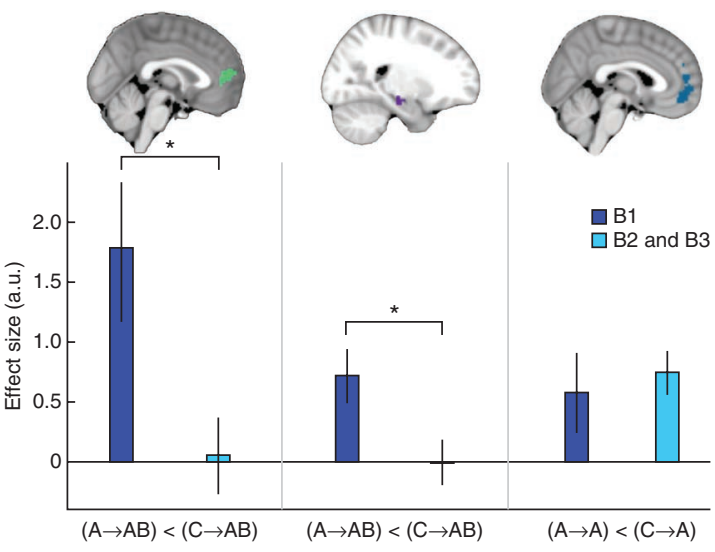

b
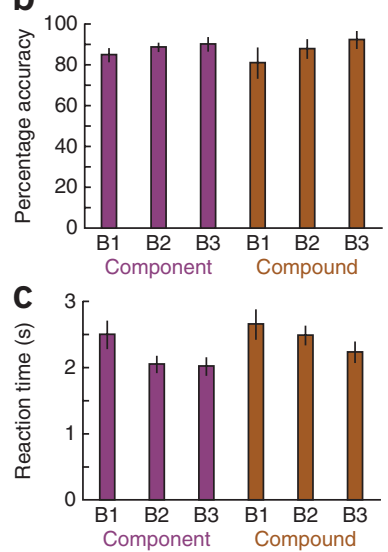

Figure 5 In the absence of sensory exposure, there was evidence for the construction mechanism only in early trials: block 1 compared with blocks 2 and 3 for unfamiliar subjects. (a) There was significantly less adaptation in blocks 2 and 3 between the novel goods and their related components in $\mathrm{mPFC}$ and hippocampus, respectively (left and middle, $P=0.024$ each; ROls are shown). There was no significant reduction across time in the $\mathrm{mPFC}$ adaptation of a component item to itself when predicted by two different stimuli (right, $P=0.326$, ROI is shown). (b,c) On the imagination task, the unfamiliar group showed an increase in accuracy (b) and a decrease in reaction time (c) across blocks. ${ }^{*} P<0.05$. Data are presented as mean \pm s.e.m. across participants. 
Figure 6 In the absence of sensory exposure, repetition suppression between related components was maintained across the duration of the experiment only if participants assigned high value to the compound goods. (a) Participants from the unfamiliar group showed significant reduction in adaptation between related components over time in mPFC, but not hippocampus ( $P=0.041$ and $P=0.785$, respectively, ROIs are shown). (b,d) The correlations shown in Figure 2 were also significant in mPFC (b) and hippocampus (d) when considering suppression effects between related components in blocks 2 and 3 alone: the amount of suppression across participants correlated positively with the average value of the compound goods (mPFC, $r=0.64, P=0.002$; hippocampus, $r=0.63$, $P=0.003$ ). (c,e) A median split of participants into those that assigned high and low values to the compound goods revealed significant suppression between related components in blocks 2 and 3 only in those participants who assigned high value (c, mPFC, High, $P=0.022 ; \mathrm{High}$ versus Low, $P=0.028$; e, hippocampus, High, $P=0.008$; High versus Low, $P<0.001$ ). ${ }^{*} P<0.05$. Data are presented as mean \pm s.e.m. across participants. to establish an independent representation of the novel good that no longer needs to be reconstructed anew on each trial.

Despite the overall reduction of cross-component suppression over the course of the experiment, this was not true for components that had been used to construct high-value novel goods. When averaging across the final two blocks, both the mPFC and hippocampus showed a significant positive correlation with the value of the compound items (mPFC, $r=0.64, P=0.002$; hippocampus, $r=0.63, P=0.003$, Fig. 6b,d and Supplementary Fig. 5), after accounting for variance explained by the value of the component items in both cases. A median split of participants according to the value assigned to the novel goods subsequently verified that there was long-lasting plasticity in $\mathrm{mPFC}$ and hippocampus in the final two blocks for those participants who attributed high, but not low, values to the novel goods (mPFC: high, $t_{8}=2.84, P=0.022$; high versus low, $t_{8}=2.68, P=0.028$; hippocampus: high, $t_{8}=3.52$, $P=0.008$; high versus low, $t_{8}=5.36, P<0.001$; Fig. 6 c,e). Suggestive evidence that value-dependent adaptation between related component items emerged later in hippocampus relative to $\mathrm{mPFC}$ (Fig. 6c,e) could not be verified statistically $\left(t_{8}=1.30, P=0.229\right)$. Together, these results suggest that the plasticity is long-lasting if value is attributed to the original association.

\section{DISCUSSION}

The role of memory in prospective evaluation and inference has been emphasized in both animals ${ }^{22}$ and humans $s^{3,4,20}$. Simulation and preplay can be used to explore an internal model of the environment and evaluate anticipated outcomes ${ }^{8,23}$. However, the neural mechanisms by which these processes are achieved have remained unclear, particularly in circumstances in which anticipated outcomes have not previously been experienced. We used repetition suppression in fMRI to reveal a neuronal mechanism that supports prospective representation and evaluation of novel experiences.

Repetition suppression has been used extensively in sensory brain regions to probe the information content of neural activations and, more recently, in more frontal brain regions, including orbitofrontal cortex ${ }^{24}$. However, a number of different hypotheses have been proposed to explain the underlying physiological mechanisms behind the phenomenon, including fatigue, sparse coding and predictive coding ${ }^{10,25-27}$. Although there is not yet a consensus on which mechanism provides the most appropriate explanation for the phenomenon, the consequences of this ambiguity are mitigated when used in a carefully controlled experimental design, as all models make the same prediction: if a neural population is sensitive to a particular feature or dimension, then suppression will occur in response to a repetition of this feature, but not others.

The repetition suppression procedure that we used was designed to allow interrogation of the underlying representation of a novel reward. By asking people to imagine and evaluate novel rewards in the scanner, we found that the neural representation of a novel reward was dependent on representations of multiple related and previously experienced rewards. Our data suggest that neuronal networks can construct a novel experience by simultaneous activation of multiple previous memories so that this constructed experience may be evaluated. Although signals in the anterior hippocampus were found to be related to construction, those in $\mathrm{mPFC}$ were related to both construction and valuation.

Crucially, unlike other goal-directed decision mechanisms that have been reported $21,23,28,29$, we only found evidence for a construction mechanism when subjects had no direct experience of an outcome, and even then only fleetingly. It is therefore possible that constructed value can provide a substitute for direct experience and train the experiential goal-directed systems that have been studied previously. This training signal may be considered analogous to off-line training of an habitual system that makes use of simulations from an internal goal-directed model ${ }^{23,30-32}$. Whereas the teaching signal provided to a habitual system replicates, or fine tunes, previous sensory experience, the teaching signal provided to a goal-directed system may establish an internal model of the future world by repeated imagination of a novel experience.

During the construction process, a second repetition suppression effect was observed between distinct and previously unassociated memories that contributed to the construction. This effect implies that the neural representation of related, compared with unrelated, component items became more similar as a consequence of the pre-scan training task, during which the participants were first exposed to the novel compounds. Notably, given that the suppression was not observed in the familiar group, it seems highly unlikely that this suppression 
effect reflects inherent similarity between related compared with unrelated components. Rather, the most plausible explanation for this change is that, through repeated representation of a novel compound, previously unrelated memories were recruited simultaneously, inducing a form of plasticity between the underlying representations of necessary components.

In both brain regions involved in construction, the $\mathrm{MPFC}$ and hippocampus, plasticity between related components was dependent on the value of the novel compounds, but not the value of components. This value dependence effect suggests that the representations of the component memories were simultaneously present during valuation of the novel compounds. A number of different mechanistic explanations may underlie this dependency. For example, the occurrence of greater blood oxygen level-dependent activity at the time of pairing may induce more plasticity or, when representing a higher value compound, the enhanced availability of neuromodulators, such as dopamine, may serve to facilitate plasticity.

Given that on average participants showed a reduction over time in the initial plasticity observed in mPFC, with comparable dynamics to the construction mechanism, it must be acknowledged that it remains ambiguous whether the adaptation observed between related components reflects classical Hebbian plasticity or even occurs in the same regions as those in which the repetition suppression is observed. However, those participants who assigned high value to the novel goods showed plasticity in mPFC that outlasted the construction process. In the hippocampus, where plasticity was not observed early on in the experiment, the same participants showed plasticity late in the experiment. Thus, the extent to which neural representations of related components became more similar to one another, but also the durability of the effect, was dependent on value attributed to the novel compounds. Irrespective of the underlying nature of the plasticity, the influence of compound value on component memories therefore supports the claim that these representations are paired together at the time of construction of the novel compounds.

The mPFC is regularly activated in studies of valuation ${ }^{17,18,33-36}$, and is particularly notable among such reward-related regions for the flexibility of the value signals that it contains. These computations may, for example, rely on an understanding of the complex structure of the environment ${ }^{5}$, the generalization of concepts learned in different situations ${ }^{3}$ or the integration of several disparate sources of information $^{37}$. If subjects are asked to ignore all of their own experiences and preferences and to instead guess what a very different individual would choose, mPFC value signals can immediately reflect the preferences of this new individual ${ }^{7,38}$. Such online evaluation is a hallmark property of goal-directed choices, which are frequently contrasted with habitual or overlearned choices in studies of animal and human behavior $6,21,23,29,39,40$. Previous studies of goal-oriented behavior have, however, focused on situations in which values are known, but must be associated with a particular course of action by inferring the structure of the world ${ }^{1,23,29}$. Our data suggest that mPFC can combine previous experiences to construct prospective outcomes de novo on each trial and can then evaluate these constructed outcomes.

Hippocampal preplay mechanisms are known to be important substrates for goal-directed spatial decisions in rodents ${ }^{8,41}$, and hippocampal value signals can be recorded in situations in which outcomes must be inferred from knowledge of relationships between stimuli in the world ${ }^{1,42}$. Notably, hippocampal activity is often recorded in concert with a network involving MPFC in studies of spatial memory and scene construction ${ }^{12,19,43}$. Consistent with the proposed function of memory in prospective inference ${ }^{44,45}$, the formation of associative links ${ }^{46,47}$ and constructive episodic simulation ${ }^{48,49}$, our data suggest that hippocampal activity can also have an active role in constructing de novo experiences in non-spatial contexts.

These findings show that a potential new experience can be prospectively represented and evaluated by invoking multiple memories simultaneously in hippocampus and mPFC. By highlighting this neuronal mechanism, we provide a unique insight into the neuronal computations underlying flexible behaviors that dominate human decision-making and that are difficult to study in animal models.

\section{METHODS}

Methods and any associated references are available in the online version of the paper.

Note: Any Supplementary Information and Source Data files are available in the online version of the paper.

\section{ACKNOWLEDGMENTS}

We thank P. Dayan, E.A. Maguire, N. Burgess, S.W. Kennerley, L.T. Hunt, M.C. Klein-Flügge and E.D. Boorman for helpful comments on an earlier draft of the manuscript, and H. Blumenthal's Fat Duck Cookbook for recipe inspiration. This study was supported by the Wellcome Trust (grant WT088312AIA to T.E.J.B. and a Senior Investigator Award to R.J.D., 098362/Z/12/Z), the Medical Research Council (grant G1000411 to H.C.B.). The Wellcome Trust Centre for Neuroimaging is supported by core funding from the Wellcome Trust Strategic Award Grant 091593/Z/10/Z.

\section{AUTHOR CONTRIBUTIONS}

All of the authors contributed to the design of the study and preparation of the manuscript. H.C.B. acquired the data and analyzed it with T.E.J.B.

\section{COMPETING FINANCIAL INTERESTS}

The authors declare no competing financial interests.

Reprints and permissions information is available online at http://www.nature.com/ reprints/index.html.

1. Wimmer, G.E. \& Shohamy, D. Preference by association: how memory mechanisms in the hippocampus bias decisions. Science 338, 270-273 (2012).

2. Peters, J. \& Büchel, C. Episodic future thinking reduces reward delay discounting through an enhancement of prefrontal-mediotemporal interactions. Neuron 66, 138-148 (2010).

3. Kumaran, D., Summerfield, J.J., Hassabis, D. \& Maguire, E.A. Tracking the emergence of conceptual knowledge during human decision making. Neuron 63, 889-901 (2009).

4. Zeithamova, D., Dominick, A.L. \& Preston, A.R. Hippocampal and ventral medial prefrontal activation during retrieval-mediated learning supports novel inference. Neuron 75, 168-179 (2012).

5. Hampton, A.N., Bossaerts, P. \& O'Doherty, J.P. The role of the ventromedial prefrontal cortex in abstract state-based inference during decision making in humans. J. Neurosci. 26, 8360-8367 (2006).

6. Gläscher, J., Daw, N., Dayan, P. \& O'Doherty, J.P. States versus rewards: dissociable neural prediction error signals underlying model-based and model-free reinforcement learning. Neuron 66, 585-595 (2010).

7. Nicolle, A. et al. An agent independent axis for executed and modeled choice in medial prefrontal cortex. Neuron 75, 1114-1121 (2012).

8. Johnson, A. \& Redish, A.D. Neural ensembles in CA3 transiently encode paths forward of the animal at a decision point. J. Neurosci. 27, 12176-12189 (2007).

9. Steiner, A.P. \& Redish, A.D. The road not taken: neural correlates of decision making in orbitofrontal cortex. Front. Neurosci. 6, 131 (2012).

10. Grill-Spector, K., Henson, R. \& Martin, A. Repetition and the brain: neural models of stimulus-specific effects. Trends Cogn. Sci. 10, 14-23 (2006).

11. Kohn, A. Visual adaptation: physiology, mechanisms and functional benefits J. Neurophysiol. 97, 3155-3164 (2007).

12. Doeller, C.F., Barry, C. \& Burgess, N. Evidence for grid cells in a human memory network. Nature 463, 657-661 (2010).

13. Kourtzi, Z. \& Kanwisher, N. Representation of perceived object shape by the human lateral occipital complex. Science 293, 1506-1509 (2001).

14. Becker, G.M., DeGroot, M.H. \& Marschak, J. Measuring utility by a single-response sequential method. Behav. Sci. 9, 226-232 (1964).

15. Kable, J.W. \& Glimcher, P.W. The neurobiology of decision: consensus and controversy. Neuron 63, 733-745 (2009).

16. Boorman, E.D., Behrens, T.E.J., Woolrich, M.W. \& Rushworth, M.F.S. How green is the grass on the other side? Frontopolar cortex and the evidence in favor of alternative courses of action. Neuron 62, 733-743 (2009). 


\section{ARTICLES}

17. Hunt, L.T. et al. Mechanisms underlying cortical activity during value-guided choice. Nat. Neurosci. 15, 470-476 (2012).

18. Jocham, G., Hunt, L.T., Near, J. \& Behrens, T.E.J. A mechanism for value-guided choice based on the excitation-inhibition balance in prefrontal cortex. Nat. Neurosci. 15, 960-961 (2012).

19. Hassabis, D., Kumaran, D. \& Maguire, E.A. Using imagination to understand the neural basis of episodic memory. J. Neurosci. 27, 14365-14374 (2007).

20. Schacter, D.L. \& Addis, D.R. Constructive memory: the ghosts of past and future. Nature 445, 27 (2007).

21. Balleine, B.W. \& Dickinson, A. Goal-directed instrumental action: contingency and incentive learning and their cortical substrates. Neuropharmacology 37, 407-419 (1998).

22. Tolman, E.C. Cognitive maps in rats and men. Psychol. Rev. 55, 189-208 (1948).

23. Daw, N.D., Gershman, S.J., Seymour, B., Dayan, P. \& Dolan, R.J. Modelbased influences on humans' choices and striatal prediction errors. Neuron 69 , 1204-1215 (2011).

24. Klein-Flügge, M.C., Barron, H.C., Brodersen, K.H., Dolan, R.J. \& Behrens, T.E.J. Segregated encoding of reward-identity and stimulus-reward associations in human orbitofrontal cortex. J. Neurosci. 33, 3202-3211 (2013).

25. Wiggs, C.L. \& Martin, A. Properties and mechanisms of perceptual priming. Curr. Opin. Neurobiol. 8, 227-233 (1998).

26. Desimone, R. Neural mechanisms for visual memory and their role in attention. Proc. Natl. Acad. Sci. USA 93, 13494-13499 (1996).

27. Summerfield, C., Trittschuh, E.H., Monti, J.M., Mesulam, M.-M. \& Egner, T. Neural repetition suppression reflects fulfilled perceptual expectations. Nat. Neurosci. 11, 1004-1006 (2008).

28. Johnson, A., Van der Meer, M.A. \& Redish, A.D. Integrating hippocampus and striatum in decision-making. Curr. Opin. Neurobiol. 17, 692-697 (2007).

29. Jones, J.L. et al. Orbitofrontal cortex supports behavior and learning using inferred but not cached values. Science 338, 953-956 (2012).

30. Sutton, R.S. Integrated architectures for learning, planning, and reacting based on approximating dynamic programming. Proc. 7th Int. Conf. Mach. Learn. 216, 224 (1990).

31. Johnson, A. \& Redish, A.D. Hippocampal replay contributes to within session learning in a temporal difference reinforcement learning model. Neural Netw. 18, 1163-1171 (2005).

32. Gershman, S.J., Markman, A.B. \& Otto, A.R. Retrospective revaluation in sequential decision making: a tale of two systems. J. Exp. Psychol. Gen. published online, doi:10.1037/a0030844 (10 December 2012) (2012).
33. Kable, J.W. \& Glimcher, P.W. The neural correlates of subjective value during intertemporal choice. Nat. Neurosci. 10, 1625-1633 (2007).

34. FitzGerald, T.H.B., Seymour, B. \& Dolan, R.J. The role of human orbitofrontal cortex in value comparison for incommensurable objects. J. Neurosci. 29, 8388-8395 (2009).

35. Plassmann, H., O'Doherty, J. \& Rangel, A. Orbitofrontal cortex encodes willingness to pay in everyday economic transactions. J. Neurosci. 27, 9984-9988 (2007).

36. Levy, I., Lazzaro, S.C., Rutledge, R.B. \& Glimcher, P.W. Choice from non-choice: predicting consumer preferences from blood oxygenation level-dependent signals obtained during passive viewing. J. Neurosci. 31, 118-125 (2011).

37. Behrens, T.E.J., Hunt, L.T., Woolrich, M.W. \& Rushworth, M.F.S. Associative learning of social value. Nature 456, 245-249 (2008).

38. Janowski, V., Camerer, C. \& Rangel, A. Empathic choice involves vmPFC value signals that are modulated by social processing implemented in IPL. Soc. Cogn. Affect. Neurosci. 8, 201-208 (2012).

39. Daw, N.D., Niv, Y. \& Dayan, P. Uncertainty-based competition between prefronta and dorsolateral striatal systems for behavioral control. Nat. Neurosci. 8 1704-1711 (2005)

40. McDannald, M.A. et al. Model-based learning and the contribution of the orbitofronta cortex to the model-free world. Eur. J. Neurosci. 35, 991-996 (2012).

41. Dragoi, G. \& Tonegawa, S. Preplay of future place cell sequences by hippocampal cellular assemblies. Nature 469, 397-401 (2011).

42. Doeller, C.F. \& Burgess, N. Distinct error-correcting and incidental learning of location relative to landmarks and boundaries. Proc. Natl. Acad. Sci. USA 105 , 5909-5914 (2008)

43. Hassabis, D. \& Maguire, E.A. Deconstructing episodic memory with construction. Trends Cogn. Sci. 11, 299-306 (2007).

44. Klein, S.B., Loftus, J. \& Kihlstrom, J.F. Memory and temporal experience: the effects of episodic memory loss on an amnesic patient's ability to remember the past and imagine the future. Soc. Cogn. 20, 353-379 (2002).

45. Buckner, R.L. The role of the hippocampus in prediction and imagination. Annu. Rev. Psychol. 61, 27-48 (2010)

46. Davachi, L. Item, context and relational episodic encoding in humans. Curr. Opin. Neurobiol. 16, 693-700 (2006).

47. Rudy, J.W. \& Sutherland, R.J. The hippocampal formation is necessary for rats to learn and remember configural discriminations. Behav. Brain Res. 34, 97-109 (1989).

48. Schacter, D.L. \& Addis, D.R. The cognitive neuroscience of constructive memory: remembering the past and imagining the future. Phil. Trans. R. Soc. Lond. B 362 , 773-786 (2007).

49. Addis, D.R., Wong, A.T. \& Schacter, D.L. Remembering the past and imagining the future: common and distinct neural substrates during event construction and elaboration. Neuropsychologia 45, 1363-1377 (2007). 


\section{ONLINE METHODS}

Participants. 39 healthy volunteers participated in the fMRI experiment and were assigned to one of two groups (unfamiliar and familiar) by drawing from Matlab's pseudo-random number generator. One participant (from the familiar group) was excluded from all further analyses because of poor performance (less than $80 \%$ accuracy on task performance during any one session). All remaining participants (19 unfamiliar participants with mean age of 28.0, 13 females, and 19 familiar participants with mean age of 27.3, 10 females) were included in further analyses with the exception of one participant (from the unfamiliar group) who was excluded from analysis of the decision task as a result of parameter estimates being more than $3.5 \mathrm{~s}$.d. away from the group mean. The final sample size was comparable to that commonly used in fMRI studies. All participants refrained from eating for $2 \mathrm{~h}$ before the start of the experiment. The study was approved by the University College London ethics committee (ref. number 3486/001) and all participants gave informed written consent.

Behavioral training. 13 different novel food combinations, or goods, were presented to the participants along with their names: tea-jelly, tomato-jam, popcorn-jelly beans, beetroot-custard, onion-mints, pea-mousse, olivestrawberry, pesto-nutella, spinach-pineapple smoothie, raspberry-avocado smoothie, vanilla-salt, yogurt-pretzels and coffee-yogurt. Each good was formed by combining two familiar component food types that had not previously been tasted together (Fig. 1a). The experimenter chose two novel goods, AB and CD, for each participant, under the constraint that the participant liked all four individual component foods ( $\mathrm{A}$ and $\mathrm{B}, \mathrm{C}$ and $\mathrm{D}$ ) from which the two novel goods were formed. All participants were given a small sample of the components (A, B, C, D) to eat, but only participants in the familiar group were allowed to taste, smell and handle the novel goods.

The experimenter randomly assigned two abstract pink shapes to each novel good $(\mathrm{AB}$ and $\mathrm{CD})$ and to their respective components (Fig. 1c). Participants were then actively trained on the 12 stimulus-item pairings using a reaction time task. On each trial, 1 of the 12 abstract shapes was shown for $400 \mathrm{~ms}$ before all six possible items were presented in randomized positions across the screen. Participants were instructed to press the button associated with the correct item as quickly and accurately as possible. Participants were required to continue with this stimulus-item learning task until their average reaction time per block approached 800 ms with $100 \%$ accuracy.

Scanning. While in the scanner, participants performed two different tasks, a decision-making task and an imagination task. The tasks were evenly divided across the three 20 -min scan sessions with 26 decision trials and 240 imagination trials in each session.

During the decision task, participants performed 78 choice trials. On each trial, photographs of two novel goods were shown on the screen and participants were given four seconds to evaluate the two options, before being prompted to indicate their preference (Fig. 1b). To encourage veridical evaluation, participants agreed to eat the chosen food from one of their decision trials (chosen at random by the computer) after exiting the scanner.

During the imagination task, an abstract pink shape was presented for $800 \mathrm{~ms}$ on each trial and this served as an instruction cue to vividly imagine the food item associated with the shape (Fig. 1d). The inter-trial interval was selected from a truncated gamma distribution with a mean of $2.5 \mathrm{~s}$. The trials were sorted into seven principal categories with 32 trials of each category per scan session, presented in a randomized order. The seven different categories were as follows: (1) novel good preceded by related component (AB preceded by A), (2) novel good preceded by unrelated component ( $\mathrm{BB}$ preceded by $\mathrm{C}$ ), (3) component preceded by the related component (A preceded by B), (4) any food item preceded by another unrelated food item in the same category, such as component preceded by unrelated component or novel good preceded by the other novel good (A preceded by $\mathrm{C}$, or $\mathrm{AB}$ preceded by $\mathrm{CD}$ ), (5) any food item (component or novel good) preceded by the same food item, but predicted by a different abstract stimulus (A preceded by A, or AB preceded by $A B$ ), (6) any food item (component or novel good) preceded by the same food item and predicted by the same abstract stimulus (A preceded by $\mathrm{A}$, or $\mathrm{AB}$ preceded by $\mathrm{AB}$ ), (7) abstract stimulus which had no association with a food outcome preceded by itself. The remaining 16 trials were necessary to ensure equal numbers of trials in each of the seven conditions.
Of particular interest for our analysis were conditions 1-4. We reasoned that neuronal assemblies that used the components to construct the novel goods would show adaptation (reduced response) in (1) relative to (2). Furthermore, if imagination of a component caused activation of the related component, we would expect reduced response in (3) relative to (4).

For each scan session, 14 yes or no questions were randomly presented during the imagination task. Each question concerned properties of the food associated with the abstract shape on the last trial; for example, 'Was the outcome salty?' Participants received $£ 0.50$ for each correct response. The adjectives used were chosen to encourage participants to elicit multisensory representations of each item, and concerned the appearance, texture, taste and smell of the food items.

Post-scan behavioral task. At the end of the scanning session, after participants had not eaten for a total of $5 \mathrm{~h}$, they were sold one of the novel goods using a BDM auction procedure ${ }^{14}$, using a previously reported protocol ${ }^{50}$. The BDM is known to elicit a measure of a participant's willingness to pay for a good ${ }^{35}$, thereby providing a measure of subjective value for each novel good.

fMRI data acquisition and pre-processing. $\mathrm{T} 2^{\star}$-weighted echo-planar images (EPIs) with blood oxygen level-dependent contrast were acquired using a 32-channel head coil on a 3Tesla Trio MRI scanner (Siemens). A special sequence was used to minimize signal drop-out in the orbitofrontal cortex (OFC) region and included an echo time (TE) of $70 \mathrm{~ms}$, a tilt of $30^{\circ}$ relative to the rostro-caudal axis and a local z-shim with a moment of $-0.4 \mathrm{mT} / \mathrm{m}$ ms applied to the OFC region. To achieve whole-brain coverage, we used 432 -mm-thick transverse slices with an inter-slice gap of $1 \mathrm{~mm}$ and in-plane resolution of $3 \times 3 \mathrm{~mm}$, and collected slices in an ascending order. This led to a repetition time of $3.01 \mathrm{~s}$. In each session, roughly 430 volumes were collected $(\sim 20 \mathrm{~min})$ and the first five volumes were discarded to allow for T1 equilibration effects. A field map with dual echo-time images (TE1 $=10 \mathrm{~ms}$, TE2 $=14.76 \mathrm{~ms}$, whole brain coverage, voxel size $3 \times 3 \times 3 \mathrm{~mm}$ ) and a single T1-weighted structural image with $1 \times 1 \times 1 \mathrm{~mm}$ voxel resolution was acquired for each participant to correct for geometric distortions and co-register the EPIs, respectively.

Preprocessing and statistical analyses were carried out using SPM8 (Wellcome Trust Centre for Neuroimaging, http://www.fil.ion.ucl.ac.uk/spm). After discarding the first five volumes, images were corrected for signal bias, realigned to the first volume, corrected for distortion using field maps, normalized to a standard EPI template and smoothed using an 8-mm full-width at half maximum Gaussian kernel.

Data analysis. Images were analyzed in an event-related manner using a general linear model (GLM) involving 32 explanatory variables. 26 explanatory variables corresponded to conditions $1-6$, which were further divided into the different food types. Four additional explanatory variables described the 'no-outcome' trials, the time of question presentation, the response to question and the time of evaluation during the decision trials. Two parametric regressors were included, corresponding to the participant's subjective value of the two novel goods and time locked to the onset the decision trials. An additional 23 nuisance regressors were included in the GLM to account for motion-related artifacts and physiological noise.

The primary aim of our analysis was to identify the neural mechanism underlying the construction of a novel good. To detect brain regions involved in evaluating the novel goods, we looked for activity modulated by chosen value during the decision task. To detect brain regions involved in constructing the novel goods (component to compound), we used the contrast [( $\mathrm{AB}$ preceded by $\mathrm{C})-(\mathrm{AB}$ preceded by $\mathrm{A})]$, averaging across all possible permutations (that is, explanatory variables (2) - (1)). To detect plasticity effects between the related components (component to component), we used the contrast [(A preceded by $\mathrm{C})-(\mathrm{A}$ preceded by $\mathrm{B})]$, again averaging across all possible permutations (that is, explanatory variables (4) - (3)). To detect brain regions showing adaptation to repeated item, but not stimulus (item to self), we used the contrast [(item preceded by different item) - (item preceded by itself but paired with a different stimulus)] (that is, explanatory variables (4) - (5)). To detect brain regions showing adaptation to repeated stimulus (stimulus adaptation), we used the contrast [(stimulus preceded by different stimulus and different item) - (stimulus preceded by itself)] (that is, explanatory variables (4) - (6)). 
The contrast images of all participants were entered into a second-level random effects analysis.

For our initial analyses, we assessed contrasts using whole-brain FWE corrected statistical significance. The cluster defining threshold was $P<0.01$ uncorrected and the corrected significance level defined as $P<0.05$. In the unfamiliar group, effects in mPFC were significant at the FWE corrected cluster level (Fig. 2a-c). To statistically assess hippocampal activity in our contrasts of interest (which did not survive cluster-based FWE thresholding), we tested the average signal from within an ROI. This ROI approach was also used to test for a difference in mPFC signal across groups and across task blocks and to test for repetition suppression effects in visual regions.

All ROIs were defined from contrasts that were orthogonal to the contrasts of interest to allow statistical tests to be performed in an unbiased fashion. To define an ROI in the hippocampi, we used the contrast identifying adaptation of 'item-to-self' averaged across all blocks (thresholded at $P<0.01$ uncorrected). A hippocampal ROI was first defined from the unfamiliar group alone (Fig. 2b) and then from the average of both groups (Figs. 4b, 5a and 6a). To assess all construction related adaptation effects in $\mathrm{MPFC}$, across groups and between blocks, we defined an ROI from the average of the two construction related contrasts (component to compound and component to component) from both groups and across all blocks (thresholded at $P<0.001$ uncorrected; Figs. 4a, 5a and 6a).

To compare the value signals in $\mathrm{mPFC}$ encoded by the two groups during the decision task, an ROI was defined from the average of the contrast for chosen value across the two groups (thresholded at $P<0.01$ uncorrected; Fig. $3 \mathbf{b}$ ). To investigate whether adaptation of component 'item-to-self' in $\mathrm{mPFC}$ reduced across the duration of the experiment, an ROI was defined using the 'item-to-self' contrast when including only component trials, and averaged across all blocks in the unfamiliar group (thresholded at $P<0.01$ uncorrected; Fig. 5a). A final ROI was defined in visual regions to test the specificity and temporal dynamics of adaptation effects, and defined from a contrast identifying a main effect to any visual event, averaged across all blocks (thresholded at $P<0.01$ uncorrected; Supplementary Fig. 4b). Note that differences between blocks or groups are, by definition, orthogonal to the group and block average effect.
The ROIs were then used to extract parameter estimates (Figs. 3c, $4 \mathbf{c}, 5 \mathbf{a}$ and $\mathbf{6 a}, \mathbf{c}, \mathbf{d})$ to test for significance between groups and across time. To assess differences between groups, we used a three-way ANOVA to test for a main effect of and an interaction between: group, unfamiliar/familiar; adaptation type, componentto-compound/component-to-component; condition, control/adaptation trial specific to the relevant adaptation type. To assess difference across time, we used a three-way ANOVA to test for a main effect of and an interaction between: block, block 1/block 2 and 3; adaptation type, component-to-compound/componentto-component; condition, control/adaptation trial specific to the relevant adaptation type. Post hoc $t$ tests were then used to decompose the results of the ANOVA, using one-tailed $t$ tests to assess changes in signal in a group, and one-tailed two sample $t$ tests to assess differences across groups. The Kolmogorov-Smirnov goodness-of-fit hypothesis test was used to check that data were approximately normally distributed.

In the unfamiliar group, first a partial correlation was performed between component-to-component suppression effects and the average value participants assigned to the two novel goods (during the BDM), after removing signal attributable to the component value (Figs. 2d,e and $\mathbf{6 b}, \mathbf{d}$ ). The adaptation signal was extracted from mPFC ROI shown in Figures $4 \mathbf{a}, \mathbf{5 a}$, and $\mathbf{6} \mathbf{a}$, and the hippocampus ROI shown in Figures 4b, 5a and 6a, averaged across all blocks (Fig. 2d,e) and then repeated using the adaptation signal from the final two blocks of trials (Fig. 6b,d). The former correlations were compared with one between adaptation effect size and average component value, using a similar partial correlation with average component value after removing effects attributable to compound value (Supplementary Fig. 1). One participant was excluded from these correlations due to missing data for the value of items. Finally, participants were divided using a median split according to high and low value attributed to the novel goods, and two-tailed $t$ tests and two-tailed paired $t$ tests used to assess adaptation in the latter two blocks between related components after variance attributable to the average component value had been removed.

50. Harris, A., Adolphs, R., Camerer, C. \& Rangel, A. Dynamic construction of stimulus values in the ventromedial prefrontal cortex. PLOS ONE 6, e21074 (2011). 